\title{
Chemical abundances and kinematics of a sample of metal-rich barium stars $\star, \star \star$
}

\author{
C. B. Pereira ${ }^{1}$, J. V. Sales Silva ${ }^{1}$, C. Chavero ${ }^{1}$, F. Roig ${ }^{1}$, and E. Jilinski ${ }^{1,2,3}$ \\ ${ }^{1}$ Observatório Nacional, Rua José Cristino, 77. CEP 20921-400, São Cristóvão, Rio de Janeiro-RJ, Brazil \\ e-mail: [claudio; joaovictor; carolina;froig;jilinski]@on.br \\ 2 Instituto de Física, Universidade do Estado do Rio de Janeiro, Rua São Francisco Xavier 524, Maracanã, \\ 200550-900 Rio de Janeiro-RJ, Brazil \\ ${ }^{3}$ Pulkovo Observatory, Russian Academy of Sciences, 65 Pulkovo, 196140 St. Petersburg, Russia
}

Received 12 April 2011 / Accepted 14 June 2011

\begin{abstract}
Aims. We determined the atmospheric parameters and abundance pattern for a sample of metal-rich barium stars. Methods. We used high-resolution optical spectroscopy. Atmospheric parameters and abundances were determined using the local thermodynamic equilibrium atmosphere models of Kurucz and the spectral analysis code MOOG.

Results. We show that the stars have enhancement factors, $[\mathrm{s} / \mathrm{Fe}]$, from 0.25 to 1.16 . Their abundance pattern of the $\mathrm{Na}, \mathrm{Al}, \alpha$ elements, and iron group elements as well as their kinematical properties are similar to the characteristics of the other metal-rich and super metal-rich stars already analyzed. We conclude that metal-rich barium stars do not belong to the bulge population. We also show that metal-rich barium stars are useful targets for probing the s-process enrichment in high-metallicity environments.
\end{abstract}

Key words. stars: abundances - stars: chemically peculiar

\section{Introduction}

Barium stars are chemically peculiar giant stars displaying both anomalously high heavy-element $(Z>30)$ abundances and an overabundance of carbon compared to the Sun, with $[\mathrm{C} / \mathrm{Fe}]$ varying from +0.4 to +1.2 (Antipova et al. 2004; Allen \& Barbuy 2006; Drake \& Pereira 2008; Pereira \& Drake 2009). In the mass-transfer hypothesis, the observed chemical peculiarities of these stars are a consequence of mass transfer through stellar winds or through Roche-lobe overflow in a binary system from an AGB star (now the white dwarf) to a less evolved companion, a barium giant or a subgiant $\mathrm{CH}$ star. Therefore, the study of their chemical peculiarities is important for understanding the physics of accretion phenomena in chemically peculiar binary systems.

Barium giants are the largest known sample among the chemically peculiar stars where the effects of the s-process nucleosynthesis can be widely investigated and measured. Busso et al. (2001) use the [hs/ls $]^{1}$ index as a function of metallicity as a criterion to probe whether the current models of s-process nucleosynthesis in AGB stars account for the observed abundance distributions.

Barium stars are found in the disk and in the halo of the Galaxy according to Gomez et al. (1997) and Mennessier et al. (1997). These two studies show that barium stars are an inhomogeneous group that can also be divided into groups according

\footnotetext{
* Based on observations made with the $1.52 \mathrm{~m}$ and $2.2 \mathrm{~m}$ telescope at the European Southern Observatory (La Silla, Chile).

$\star \star$ Tables 2 and 4 are only available at the CDS via anonymous ftp to cdsarc.u-strasbg.fr $(130.79 .128 .5)$ or via

http://cdsarc.u-strasbg.fr/viz-bin/qcat?]/A+A/533/A51

1 [hs $/ \mathrm{ls}]=\log (\mathrm{hs} / \mathrm{ls})_{\star}-\log (\mathrm{hs} / \mathrm{ls})_{\odot}$ where $[\mathrm{hs}]$ and $[\mathrm{ls}]$ are the mean abundances of the s-elements at the $\mathrm{Ba}$ and $\mathrm{Zr}$ peaks, respectively.
}

to their luminosities, kinematical and spatial parameters $\left(U_{0}\right.$, $V_{0}, W_{0}$ velocities and dispersion velocities and scale heights). If the barium star phenomenon is seen both in the disk and in the halo, they are very useful objects to probe the s-process nucleosynthesis at different metallicities, in different population. Barium stars have already been analyzed in the halo, such as HD 206983 (Junqueira \& Pereira 2001; Drake \& Pereira 2008), HD 10613 (Pereira \& Drake 2009), and HD 123396 (Allen \& Barbuy 2006), and their connection with the metal-poor halo yellow symbiotic stars have already been investigated (Jorissen et al. 2005; Pereira \& Drake 2009). Therefore, the quantitatively confirmation of the overabundances of the s-process elements in a sample of barium stars would help to better constrain the number of known barium stars, which it would be useful to compare with their theoretical birthrate (Han et al. 1995). Motivated by the questions mentioned above, we started a high-resolution spectroscopic survey of the barium stars from the samples of MacConnell et al. (1972) and Bidelman (1981) as well as some stars from Gomez et al. (1997). As a first result of 230 surveyed stars, we have already discovered a new $\mathrm{CH}$ subgiant, $\mathrm{BD}-$ $03^{\circ} 3668$ (Pereira \& Drake 2011).

In this paper we report another result from our survey, that is, the discovery of a small sample of 12 metal-rich $([\mathrm{Fe} / \mathrm{H}] \geq$ +0.1 ) barium stars. According to their metallicities, metal-rich stars can be divided into two sub-samples (Grenon 1972). Stars with metallicities $0.08<[\mathrm{Fe} / \mathrm{H}]<+0.2$ are the metal-rich ones and those with metallicities $+0.2<[\mathrm{Fe} / \mathrm{H}]<+0.5$ are the super metal-rich. According to this scheme, we found 7 metal-rich and 5 super metal-rich barium stars. The "very metal-rich" stars were first identified by Arp (1965) investigating the nucleus of the galaxy NGC 6522. Arp (1965) also derived their age to be between $10^{9}$ to $10^{10}$ Gyr. Spinrad \& Taylor (1969) surveyed a large group of $\mathrm{K}$ giants with metal abundances greater than that of 
the Hyades and with abundance variations among their sample. These authors also found the giants ages to be older than the disk star, Grenon (1999) also showed that metal-rich stars have an age mixture ranging from $0.7 \mathrm{Gyr}$ for the Hyades generation to an intermediate generation of 3-4 Gyr and to an oldest generation, 10 Gyr. In the investigation of the photometric and kinematic properties of a large sample of G and K giants, Eggen (1993) found that $10 \%$ of the stars in the solar neighborhood have metallicities higher than +0.15 dex. Eggen (1993) also identified a sample of barium stars among the evolved stars in the old disk population that could be metal-rich as well. However, none of the stars presented in Table 11 of his paper can be classified as metal-rich or super metal rich (HD 46407, $[\mathrm{Fe} / \mathrm{H}]=-0.14$, Antipova et al. (2003); HD 116713 and HD 202109 with respectively $[\mathrm{Fe} / \mathrm{H}]=-0.12$ and -0.04 , Smiljanic et al. (2007); HD $83548,[\mathrm{Fe} / \mathrm{H}]=+0.03$, Pereira et al. (in prep.) ; and NGC 2420$\mathrm{D},[\mathrm{Fe} / \mathrm{H}]=-0.55$, Smith \& Suntzeff (1987)). Metal-rich stars have a mean distance from the Galactic plane of $0.2 \mathrm{kpc}$ and and their Galactic orbits appear to be located inside the solar Galactic orbit (Grenon 1999). Metal-rich stars in the solar neighborhood either could be diffused into the disk from the bulge population (Barbuy \& Grenon 1990) or they may be the representatives of the last stages of the chemical evolution of the disk (Matteucci 2003).

We here analyze the high-resolution spectra of a sample of metal-rich barium stars to obtain $[\mathrm{X} / \mathrm{Fe}]$ versus $[\mathrm{Fe} / \mathrm{H}]$ trends and also to investigate the heavy-element abundance pattern in metal-rich environments. As we shall see, all our stars have spectral types $\mathrm{G}$ and $\mathrm{K}$, and are accordingly free from the strong molecular opacity from $\mathrm{ZrO}, \mathrm{CN}$ and $\mathrm{C}_{2}$ absorption features, which complicates a quantitative analysis and the measurement of some atomic lines. These molecules are usually observed in MS, S, and disk carbon stars at near solar metallicity where enhancements from s-process nucleosynthesis have already been reported (Busso et al. 2001).

\section{Observations}

The high-resolution spectra of our stars were obtained with the FEROS (Fiberfed Extended Range Optical Spectrograph) Echelle spectrograph (Kaufer et al. 1999) at the $1.52 \mathrm{~m}$ and $2.2 \mathrm{~m}$ ESO telescopes at La Silla (Chile) in several runs between 2000 and 2010. The FEROS spectral resolving power is $R=48000$, corresponding to 2.2 pixels of $15 \mu \mathrm{m}$, and the wavelength coverage goes from $3800 \AA$ to $9200 \AA$. The nominal $S / N$ ratio was evaluated by measuring the rms flux fluctuation in selected continuum windows, and the typical values were $S / N=100-200$. The spectra were reduced with the MIDAS pipeline reduction package consisting of the following standard steps: CCD bias correction, flat-fielding, spectrum extraction, wavelength calibration, correction of barycentric velocity, and spectrum rectification. Table 1 shows the log of observations and some previous information ( $V$-magnitude, spectral types and the literature sources from where the stars were selected) of the studied stars.

\section{Analysis and results}

\subsection{Line selection, measurements, and oscillator strengths}

Several atomic absorption lines used in this study are the same as were used in previous studies dedicated to the analysis of photospheric abundances of the barium stars (Pereira 2005; Pereira \& Drake 2009, 2011). The chosen lines are sufficiently unblended to yield reliable abundances. Table 2 shows the Fe I and Fe II lines

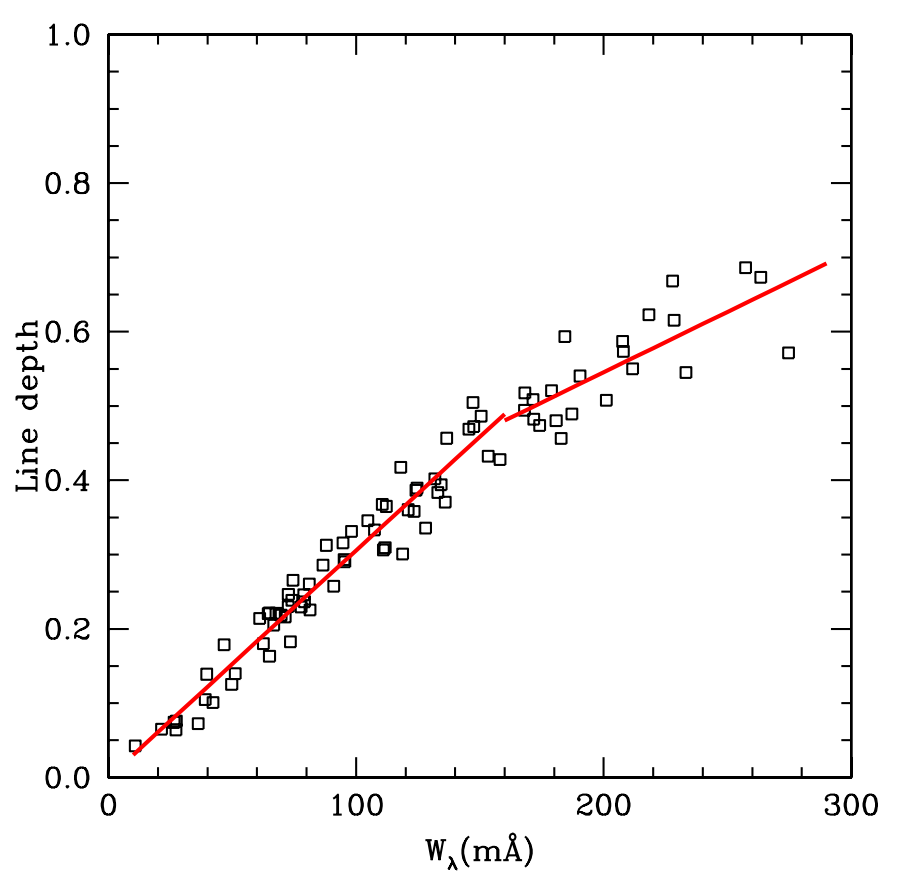

Fig. 1. Line depth vs. equivalent width of $\mathrm{Fe}_{\mathrm{I}}$ lines of CD-25 6606. This plot shows that there is a change in the slope around $160 \mathrm{~m} \AA$ which was used to set this limit for the equivalent widths in our abundance analysis.

employed in the analysis and also the lower excitation potential, $\chi(\mathrm{eV})$, of the transitions, the $\log g f$ values, and the measured equivalent widths. The latter were obtained by fitting Gaussian profiles to the observed ones. The $\log g f$ values for the Fe I and Fe II lines were taken from Lambert et al. (1996) and Castro et al. (1997).

Absorption lines whose equivalent widths are not stronger than $150 \mathrm{~m} \AA$ should lie in the linear part of the curve of growth (Hill et al. 1995). We can evaluate if this limit is also valid for our measurements of equivalent widths by analyzing a plot of line depth vs. equivalent width. In this kind of plot we aim to examine whether (and where) there would be a significant change in the behavior of the absorption lines when the weaker and stronger lines are studied together. Figure 1 shows these measurements of several Fe I lines in the spectrum of CD-25 6606. We fitted the data using two linear functions, one between $0.0 \mathrm{~m} \AA$ and $W_{\text {turn }}$, and another between $W_{\text {turn }}$ and $300 \mathrm{~m} \AA$. By varying the value of $W_{\text {turn }}$ between 100 and $200 \mathrm{~m} \AA$ we verified that the best fits for the two linear functions simultaneously hold for $W_{\text {turn }} \sim 160-170 \mathrm{~m} \AA$. At this point, the slope shows a reduction of about $50 \%$ (from $\sim 0.0031$ to $\sim 0.0017$ ). Figure 1 also shows that up to $\sim 160 \mathrm{~m} \AA$ the $\chi$-square of fit is lower (0.0008) than the $\chi$-square between 160 and $300 \mathrm{~m} \AA$, which is 0.002 . The change of slope and the large dispersion upward of $160 \mathrm{~m} \AA$ are caused by saturation effects that become important for the stronger lines so that the Gaussian profiles would not be the best representation for the line profiles. Therefore, lines with equivalent widths stronger than $160 \mathrm{~m} \AA$ were not used in our abundance analysis for the stars analyzed in this work. For the same reason we did not use any of the barium lines in our sample stars. 
Table 1. Log of the observations and basic information of the stars.

\begin{tabular}{|c|c|c|c|c|c|}
\hline$\overline{\text { Star }}$ & Date & $\begin{array}{c}\text { Exposure time } \\
\text { (s) }\end{array}$ & $\overline{\bar{V} \text { (mag) }}$ & $\overline{\text { SpT }^{a}}$ & Source \\
\hline CD-256606 & $22 / 02 / 2008$ & 1200 & 9.3 & - & $\mathrm{II}^{b}$ \\
\hline HD 46040 & $14 / 10 / 2008$ & 420 & 8.0 & K0p & $\mathrm{I}^{c}$ \\
\hline HD 49841 & $27 / 01 / 2001$ & 3600 & 9.0 & G5 & I \\
\hline HD 82765 & $17 / 02 / 2008$ & 600 & 8.5 & G8 III & II \\
\hline HD 84734 & $17 / 02 / 2008$ & 600 & 9.0 & - & II \\
\hline HD 85205 & $25 / 03 / 2000$ & 720 & 8.4 & G6 II & II \\
\hline HD 100012 & $13 / 02 / 2008$ & 420 & 6.6 & K0/K1 III & II \\
\hline HD 101079 & $03 / 04 / 2007$ & 600 & 8.2 & $\mathrm{~K} 2$ & $\mathrm{~B} 81^{d}$ \\
\hline HD 130386 & $28 / 07 / 2009$ & 420 & 7.8 & $\mathrm{~K} 2$ & II \\
\hline HD 139660 & 03/04/2007 & 900 & 8.4 & K0 & $\mathrm{G} 97^{e}$ \\
\hline HD 198590 & $18 / 08 / 2008$ & 360 & 7.0 & G8 II & II \\
\hline HD 212209 & $18 / 08 / 2008$ & 600 & 8.9 & - & II \\
\hline
\end{tabular}

References. ${ }^{(a)}$ Gomez et al. (1997); ${ }^{(b)}$ Table II of MacConnell et al. (1972); ${ }^{(c)}$ Table I of MacConnell et al. (1972); ${ }^{(d)}$ Bidelman (1981); ${ }^{(e)}$ Gomez et al. (1997).

\subsection{Determination of the atmospheric parameters}

The determination of stellar atmospheric parameters effective temperature $\left(\mathrm{T}_{\mathrm{eff}}\right)$, surface gravity $(\log g)$, microturbulence $(\xi)$, and $[\mathrm{Fe} / \mathrm{H}]$ (throughout, we use the notation $[\mathrm{X} / \mathrm{H}]=$ $\left.\log (N(\mathrm{X}) / N(\mathrm{H}))_{\star}-\log \left(N(\mathrm{X}) / N(\mathrm{H})_{\odot}\right)\right)$ are prerequisites for the determination of photospheric abundances. The atmospheric parameters were determined by assuming local thermodynamic equilibrium (hereafter LTE) model atmospheres of Kurucz (1993) using the spectral analysis code MOOG (Sneden 1973).

The solution of the excitation equilibrium used to derive the temperature ( $\left.T_{\text {eff }}\right)$ was defined by the zero slope of the trend between the iron abundances derived from FeI lines and the excitation potential of the measured lines. The microturbulent velocity $(\xi)$ was determined by constraining the abundance determined from individual $\mathrm{Fe}_{\mathrm{I}}$ lines to show no dependence on $W_{\lambda} / \lambda$. The solution thus found is unique, depending only on the set of $\mathrm{Fe}$ I,II lines and the atmospheric model employed, and yields as a by-product the metallicity of the star $[\mathrm{Fe} / \mathrm{H}]$. The final adopted atmospheric parameters are given in Table 3 . The value of $\log$ $g$ seen in Table 3 was determined by means of the ionization balance using the assumption of LTE. We found typical uncertainties of $\sigma\left(T_{\text {eff }}\right)=150 \mathrm{~K}, \sigma(\log g)=0.2 \mathrm{dex}$, and $\sigma(\xi)=$ $0.2 \mathrm{~km} \mathrm{~s}^{-1}$.

Previous determinations of atmospheric parameters of two stars of our sample, HD 100012 and HD 130386, were made by Luck \& Bond (1991) and Antipova et al. (2004), respectively. Luck \& Bond (1991) found for HD 100012, $T_{\text {eff }}=5000 \mathrm{~K}$, $\log g=2.3 \mathrm{dex}, V_{\mathrm{t}}=2.2 \mathrm{~km} \mathrm{~s}^{-1}$ and $[\mathrm{Fe} / \mathrm{H}]=+0.33$, while for HD 130386, Antipova et al. (2004) found $T_{\mathrm{eff}}=4720 \mathrm{~K}$, $\log g=2.4 \operatorname{dex}, V_{\mathrm{t}}=1.4 \mathrm{~km} \mathrm{~s}^{-1}$ and $[\mathrm{Fe} / \mathrm{H}]=0.01 \mathrm{dex}$.

\subsection{Abundance analysis}

The abundances of chemical elements were determined with the LTE model atmosphere techniques. In brief, equivalent widths are calculated by integration through a model atmosphere and are compared with the observed equivalent widths. The calculations are repeated, changing the abundance of the element in question, until a match is achieved. The current version of the line-synthesis code MOoG (Sneden 1973) was used to carry out the calculations. Table 4 shows the atomic lines used to derive the abundances of the elements. Atomic parameters for several transitions of $\mathrm{Ti}, \mathrm{Cr}$, and $\mathrm{Ni}$ were retrieved from the library of the National Institute of Science and Technology Atomic Spectra
Database (Martin et al. 2002). Tables 5 and 6 provide the derived abundances in the notation $[\mathrm{X} / \mathrm{Fe}]$. The last two columns of Table 6 give the mean abundance of the s-process elements and their standard deviations, i.e., the scatter around the mean.

\subsection{Abundance uncertainties}

The uncertainties of the derived abundances for the program stars are dominated by two main sources: the errors in the stellar parameters and errors in the equivalent width measurements.

The abundance uncertainties owing to the errors in the stellar atmospheric parameters $T_{\text {eff }}, \log g$, and $\xi$ were estimated by changing these parameters by their standard errors and then computing the changes incurred in the element abundances. This technique was applied in the abundances determined from equivalent line widths. The results of these calculations for CD$25^{\circ} 6606$ are displayed in Cols. 2 to 5 of Table 7 . The abundance variations for the other stars show similar values.

The abundance uncertainties owing to the errors in the equivalent widths measurements were computed from an expression provided by Cayrel (1988). The errors in the equivalent widths are set, essentially, by the signal-to-noise ratio and the resolution of the spectra. In our case, having $R \approx 50000$ and typical $S / N$ of 150 , the expected uncertainties in the equivalent widths are about 2-3 m $\AA$. For all measured equivalent widths, these uncertainties lead to the errors in the abundances less than those from the sum of the uncertainties owing to the stellar parameters.

Under the assumption that the errors are independent, they can be combined quadratically so that the total uncertainty is

$$
\sigma=\sqrt{\sum_{i=1}^{N} \sigma_{i}^{2}}
$$

These final uncertainties are given in the sixth column of Table 7. The last column gives the observed abundance dispersion among the lines for those elements with more than three available lines. Table 7 also shows that neutral elements are fairly sensitive to the temperature variations, while single ionized elements are sensitive to the $\log g$ variations. The uncertainties on microturbulence also contribute to the compounded errors. 
Table 3. Atmospheric parameters, radial velocities, and galactic latitude.

\begin{tabular}{lccccccc}
\hline \hline & $T_{\text {eff }}(\mathrm{K})$ & $\log g$ & {$[\mathrm{FeI} / \mathrm{H}]$} & {$[\mathrm{FeII} / \mathrm{H}]$} & $\xi\left(\mathrm{km} \mathrm{s}^{-1}\right)$ & $V_{r}\left(\mathrm{~km} \mathrm{~s}^{-1}\right)$ & latitude gal. $\left(b^{\circ}\right)$ \\
\hline CD-25 6606 & 5300 & 2.7 & $+0.12 \pm 0.14(55)$ & $+0.10 \pm 0.09(10)$ & 1.5 & $+0.99 \pm 0.91$ & +11.04 \\
HD 46040 & 4800 & 2.4 & $+0.11 \pm 0.13(27)$ & $+0.11 \pm 0.14(9)$ & 1.4 & $+23.72 \pm 0.33$ & -29.59 \\
HD 49841 & 5200 & 3.2 & $+0.21 \pm 0.13(55)$ & $+0.20 \pm 0.19(12)$ & 1.3 & $+8.17 \pm 0.50$ & +02.40 \\
HD 82765 & 5100 & 2.6 & $+0.19 \pm 0.10(43)$ & $+0.20 \pm 0.11(11)$ & 1.4 & $+10.53 \pm 0.49$ & -10.65 \\
HD 84734 & 5200 & 2.9 & $+0.20 \pm 0.12(40)$ & $+0.21 \pm 0.11(11)$ & 1.5 & $-27.38 \pm 2.81$ & -11.68 \\
HD 85205 & 5300 & 2.8 & $+0.23 \pm 0.16(41)$ & $+0.24 \pm 0.09(12)$ & 1.9 & $+25.31 \pm 0.58$ & +13.70 \\
HD 100012 & 5000 & 2.7 & $+0.18 \pm 0.12(45)$ & $+0.19 \pm 0.10(11)$ & 1.5 & $+8.95 \pm 0.65$ & +33.59 \\
HD 101079 & 5000 & 2.7 & $+0.10 \pm 0.12(52)$ & $+0.09 \pm 0.08(11)$ & 1.3 & $-2.77 \pm 0.55$ & +56.64 \\
HD 130386 & 4900 & 2.7 & $+0.16 \pm 0.13(39)$ & $+0.14 \pm 0.11(10)$ & 1.3 & $+16.46 \pm 0.45$ & +46.88 \\
HD 139660 & 5000 & 2.8 & $+0.26 \pm 0.14(44)$ & $+0.26 \pm 0.11(12)$ & 1.4 & $-13.22 \pm 0.54$ & +35.11 \\
HD 198590 & 5100 & 2.6 & $+0.18 \pm 0.14(48)$ & $+0.17 \pm 0.13(11)$ & 1.5 & $-9.62 \pm 0.42$ & -39.51 \\
HD 212209 & 4700 & 2.4 & $+0.30 \pm 0.13(38)$ & $+0.30 \pm 0.13(10)$ & 1.2 & $-27.23 \pm 0.52$ & -48.29 \\
\hline
\end{tabular}

Table 5. Abundance ratios $[\mathrm{X} / \mathrm{Fe}]$ for the elements from $\mathrm{Na}$ to $\mathrm{Ni}$.

\begin{tabular}{lcccccccc}
\hline \hline Star & {$[\mathrm{Na} / \mathrm{Fe}]$} & {$[\mathrm{Mg} / \mathrm{Fe}]$} & {$[\mathrm{Al} / \mathrm{Fe}]$} & {$[\mathrm{Si} / \mathrm{Fe}]$} & {$[\mathrm{Ca} / \mathrm{Fe}]$} & {$[\mathrm{Ti} / \mathrm{Fe}]$} & {$[\mathrm{Cr} / \mathrm{Fe}]$} & {$[\mathrm{Ni} / \mathrm{Fe}]$} \\
\hline CD-25 6606 & +0.19 & +0.05 & -0.02 & +0.11 & +0.08 & -0.04 & +0.03 & 0.00 \\
HD 46040 & +0.16 & +0.04 & +0.02 & -0.09 & +0.11 & +0.03 & +0.06 & -0.04 \\
HD 49841 & +0.04 & -0.08 & -0.11 & -0.03 & +0.07 & -0.01 & 0.00 & +0.02 \\
HD 82765 & +0.15 & +0.18 & +0.06 & +0.15 & +0.14 & 0.00 & +0.06 & +0.08 \\
HD 84734 & +0.12 & +0.19 & -0.08 & +0.21 & +0.06 & +0.01 & +0.05 & +0.03 \\
HD 85205 & +0.23 & +0.11 & +0.11 & +0.09 & +0.08 & -0.05 & -0.01 & +0.08 \\
HD 100012 & +0.29 & +0.19 & 0.00 & +0.15 & +0.05 & +0.04 & 0.00 & +0.06 \\
HD 101079 & +0.20 & +0.14 & +0.23 & +0.19 & +0.10 & -0.06 & +0.02 & +0.03 \\
HD 130386 & +0.36 & +0.19 & +0.21 & +0.19 & +0.15 & +0.09 & +0.03 & +0.09 \\
HD 139660 & +0.23 & +0.06 & +0.07 & +0.19 & +0.07 & -0.05 & +0.03 & +0.05 \\
HD 198590 & +0.21 & +0.20 & +0.16 & +0.22 & +0.10 & -0.09 & -0.02 & +0.03 \\
HD 212209 & +0.15 & +0.12 & +0.18 & +0.20 & +0.06 & -0.03 & 0.00 & +0.07 \\
\hline
\end{tabular}

Table 6. Abundance ratios $[\mathrm{X} / \mathrm{Fe}]$ for the heavy elements.

\begin{tabular}{lccccccc}
\hline \hline Star & {$[\mathrm{Y} / \mathrm{Fe}]$} & {$[\mathrm{Zr} / \mathrm{Fe}]$} & {$[\mathrm{La} / \mathrm{Fe}]$} & {$[\mathrm{Ce} / \mathrm{Fe}]$} & {$[\mathrm{Nd} / \mathrm{Fe}]$} & {$[\mathrm{s} / \mathrm{Fe}]$} & $\sigma([\mathrm{s} / \mathrm{Fe}])$ \\
\hline CD-25 6606 & +0.62 & +0.48 & +0.73 & +0.52 & +0.85 & +0.64 & 0.15 \\
HD 46040 & +0.93 & +1.04 & +1.83 & +1.07 & +0.94 & +1.16 & 0.38 \\
HD 49841 & +0.85 & +0.65 & +0.81 & +0.56 & +0.92 & +0.76 & 0.15 \\
HD 82765 & +0.52 & +0.37 & +0.35 & +0.12 & +0.20 & +0.31 & 0.14 \\
HD 84734 & +0.64 & +0.60 & +0.74 & +0.56 & +0.80 & +0.67 & 0.10 \\
HD 85205 & +0.65 & +0.65 & +0.58 & +0.44 & +0.80 & +0.62 & 0.14 \\
HD 100012 & -0.03 & +0.13 & +0.29 & +0.25 & +0.45 & +0.22 & 0.18 \\
HD 101079 & +0.63 & +0.44 & +0.43 & +0.28 & +0.51 & +0.46 & 0.13 \\
HD 130386 & +0.65 & +0.59 & +0.47 & +0.47 & +0.29 & +0.49 & 0.14 \\
HD 139660 & +0.77 & +0.51 & +0.56 & +0.35 & +0.50 & +0.54 & 0.15 \\
HD 198590 & +0.69 & +0.51 & +0.38 & +0.33 & +0.33 & +0.45 & 0.15 \\
HD 212209 & +0.53 & +0.24 & +0.24 & +0.06 & +0.18 & +0.25 & 0.17 \\
\hline
\end{tabular}

\section{Discussion}

\subsection{The position of the stars in the $\log g-\log T_{\text {eff }}$ diagram}

Figure 2 shows the position of the studied stars in the $\log T_{\text {eff }}-$ $\log g$ diagram, where model tracks were computed by Schaerer et al. (1993) for the stars of 2.0, 2.5, and 3.0 solar masses with metallicity of $Z=0.04$. Putting the studied stars in this diagram, we estimated the masses as given in Table 8 . Table 8 also provides the distance that resulted from the assumed mass from the $\log T_{\text {eff }}-\log g$ diagram and from the temperature and surface gravity given in Table 3. In these calculations we assumed the bolometric corrections of Alonso et al. (1999) and $M_{\text {bol } \odot}=+4.74$ (Bessell et al. 1998). The last column of Table 8 provides the distance of the stars whose parallax were measured by Hipparcos. The distances were derived based on the ionization equilibrium and the derived masses from the positions in the $\log g-\log T_{\text {eff }}$ diagram, and astrometric values agree well within the uncertainties with the possible exception of HD 13966.

The stars CD-256606, HD 49841, HD 84734, and HD 85205 , with $\log T_{\text {eff }}=3.72$ and $\log g=2.7,3.2,2.9$, and 2.8 respectively, occupy a region that is at the end of subgiant phase or near the base of red giant branch in the $\log g-\log T_{\text {eff }}$ diagram. This is important because in all high-resolution spectroscopy studies dedicated to the analyses of the spectra of barium dwarfs and $\mathrm{CH}$ subgiant stars, no stars of near solar metallicity or even higher than the solar metallicity in this evolutionary phase were found (Smith et al. 1993; Pereira 2005; Allen \& Barbuy 2006). Therefore, these four stars might be the first identified metal-rich $\mathrm{CH}$ subgiants. Barium dwarfs $(\log g \geq 4.0)$ with metallicities close to the solar one are as yet undiscovered (Pereira \& Drake 2011). 
Table 7. Abundance uncertainties for CD-25 6606.

\begin{tabular}{lcccccc}
\hline \hline Species & $\begin{array}{c}\Delta T_{\text {eff }} \\
+150 \mathrm{~K}\end{array}$ & $\begin{array}{c}\Delta \log g \\
+0.3\end{array}$ & $\begin{array}{c}\Delta \xi \\
+0.3\end{array}$ & $\begin{array}{c}\Delta W_{\lambda} \\
+3 \mathrm{~m} \AA\end{array}$ & $\left(\sum \sigma^{2}\right)^{1 / 2}$ & $\sigma_{\text {obs }}$ \\
& +0.12 & 0.00 & -0.13 & +0.07 & 0.19 & 0.14 \\
\hline $\mathrm{Fe}_{\text {I }}$ & +0.09 & +0.11 & -0.14 & +0.07 & 0.21 & 0.09 \\
$\mathrm{Fe}_{\text {II }}$ & +0.10 & -0.01 & -0.06 & +0.06 & 0.13 & 0.15 \\
$\mathrm{Na}_{\text {I }}$ & +0.05 & -0.03 & -0.06 & +0.04 & 0.09 & 0.18 \\
$\mathrm{Mg}_{\text {I }}$ & +0.07 & -0.02 & -0.05 & +0.05 & 0.10 & 0.19 \\
$\mathrm{Al}_{\text {I }}$ & +0.01 & +0.02 & -0.05 & +0.06 & 0.08 & 0.06 \\
$\mathrm{Si}_{\text {I }}$ & +0.13 & -0.01 & -0.14 & +0.07 & 0.20 & 0.10 \\
$\mathrm{Ca}_{\text {I }}$ & +0.28 & +0.01 & -0.07 & +0.07 & 0.29 & 0.09 \\
$\mathrm{Ti}_{\text {I }}$ & +0.15 & +0.02 & -0.09 & +0.08 & 0.19 & 0.18 \\
$\mathrm{Cr}_{\text {I }}$ & +0.11 & +0.03 & -0.10 & +0.07 & 0.17 & 0.12 \\
$\mathrm{Ni}_{\text {I }}$ & +0.01 & +0.12 & -0.16 & +0.08 & 0.22 & 0.06 \\
$\mathrm{Y}_{\text {II }}$ & +0.21 & +0.02 & -0.01 & +0.08 & 0.23 & 0.04 \\
$\mathrm{Zr}_{\text {I }}$ & +0.02 & +0.13 & -0.06 & +0.07 & 0.16 & 0.19 \\
$\mathrm{La}_{\text {II }}$ & +0.02 & +0.13 & -0.09 & +0.08 & 0.18 & 0.11 \\
$\mathrm{Ce}_{\text {II }}$ & +0.04 & +0.15 & -0.15 & +0.10 & 0.24 & 0.14 \\
$\mathrm{Nd}_{\text {II }}$ & & & & & & \\
\hline & & &
\end{tabular}

Notes. The second column gives the variation of the abundance caused by the variation in $T_{\text {eff }}$. The other columns refer to the variations caused by $\log g, \xi$, and $W_{\lambda}$, respectively. The sixth column gives the compounded r.m.s. uncertainty of the second to fifth columns. The last column gives the observed abundance dispersion for those elements whose abundances were derived using more than three lines.

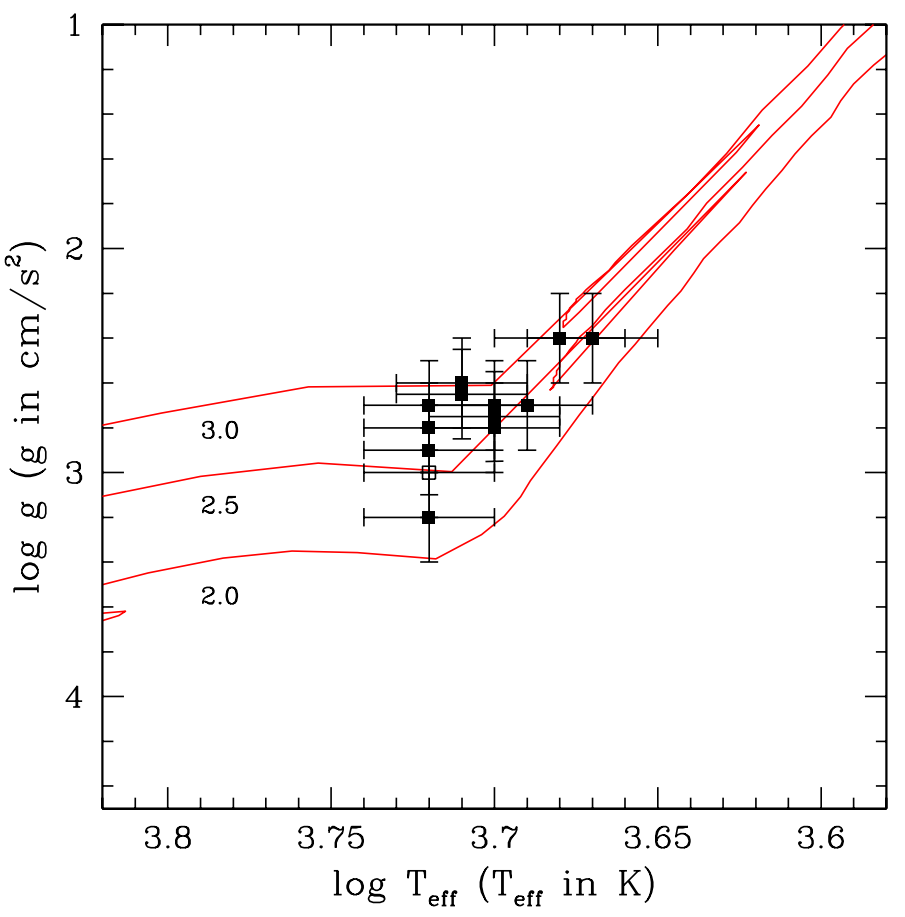

Fig. 2. Position of the stars (filled squares) in the $\log g-\log T_{\text {eff }}$ diagram. Evolutionary tracks are from Schaerer et al. (1993). The numbers correspond to stellar masses in units of solar mass. Red lines represent evolutionary tracks for a star with $Z=0.04$. The stars CD-25 6606, HD 49841, HD 84734, and HD 85205 are subgiant stars about to begin their ascent of the red giant branch, like S 190, a D'-type symbiotic star (open square) that is also barium enriched (Smith et al. 2001).

\subsection{Kinematics}

As we mentioned in the introduction, some metal-rich stars could be old objects. Figure 3 shows the position of stars analyzed in this work in the HR-diagram with the isochrones of Girardi et al. (2000). The metal-rich barium stars with ages
Table 8. Masses and distances of the stars.

\begin{tabular}{lccc}
\hline \hline Star & $M / M_{\odot}$ & $r(\mathrm{pc})$ & $r(\mathrm{pc})($ Hipparcos $)$ \\
\hline CD-25 6606 & 3.0 & $820 \pm 200$ & - \\
HD 46040 & 2.5 & $440 \pm 100$ & $320 \pm 70$ \\
HD 49841 & 2.0 & $310 \pm 70$ & - \\
HD 82765 & 2.5 & $520 \pm 120$ & $420 \pm 140$ \\
HD 84734 & 2.5 & $490 \pm 115$ & - \\
HD 85205 & 2.5 & $440 \pm 100$ & - \\
HD 100012 & 2.5 & $190 \pm 45$ & $180 \pm 25$ \\
HD 101079 & 2.5 & $390 \pm 90$ & - \\
HD 130386 & 2.5 & $300 \pm 70$ & $290 \pm 110$ \\
HD 139660 & 2.5 & $380 \pm 90$ & $190 \pm 44$ \\
HD 198590 & 2.5 & $260 \pm 60$ & $410 \pm 140$ \\
HD 212209 & 2.5 & $630 \pm 150$ & $390 \pm 180$ \\
\hline
\end{tabular}

between 8.5 and 9.0 Gyr are similar to the metal-rich stars analyzed by Pakhomov et al. (2009), which have a mean age of $\approx 8.7$ Gyr. Our results confirm the results of Mennessier et al. (1997) that some barium stars in this range of gravity (and also in absolute magnitudes) have ages between 8.5 and 9.5 Gyr.

We also investigated the kinematical properties of our sample stars. Table 9 shows our results. The radial velocities were determined by measuring the Doppler shift of the spectral lines and are given in Table 3. Distances and proper motions were taken from the Hipparcos catalog (Perryman \& ESA 1997). Space velocities $\left(U_{\odot}, V_{\odot}, W_{\odot}\right)$ relative to the local standard of rest were computed based on the method of Johnson \& Soderblom (1987) and were calculated assuming the solar motion of $(U, V, W)=(11.1,12.2,7.3) \mathrm{km} \mathrm{s}^{-1}$, as derived by Schönrich et al. (2010). The Galactic orbital parameters $R_{\min }$ and $R_{\max }$ (minimum and maximum distances from the Galactic center), $Z_{\max }$ (maximum distance from Galactic plane) and $e$ (orbital eccentricity) were obtained using the Galactic potential integrator developed by C. Flynn (http://www . astro .utu.fi/ galorb.html). For this we adopted a solar Galactocentric distance of $8.5 \mathrm{kpc}$ and circular velocity of $220 \mathrm{~km} \mathrm{~s}^{-1}$. Because barium stars are binary stars, their observed radial velocities are affected by periodical variations. If we have only one 


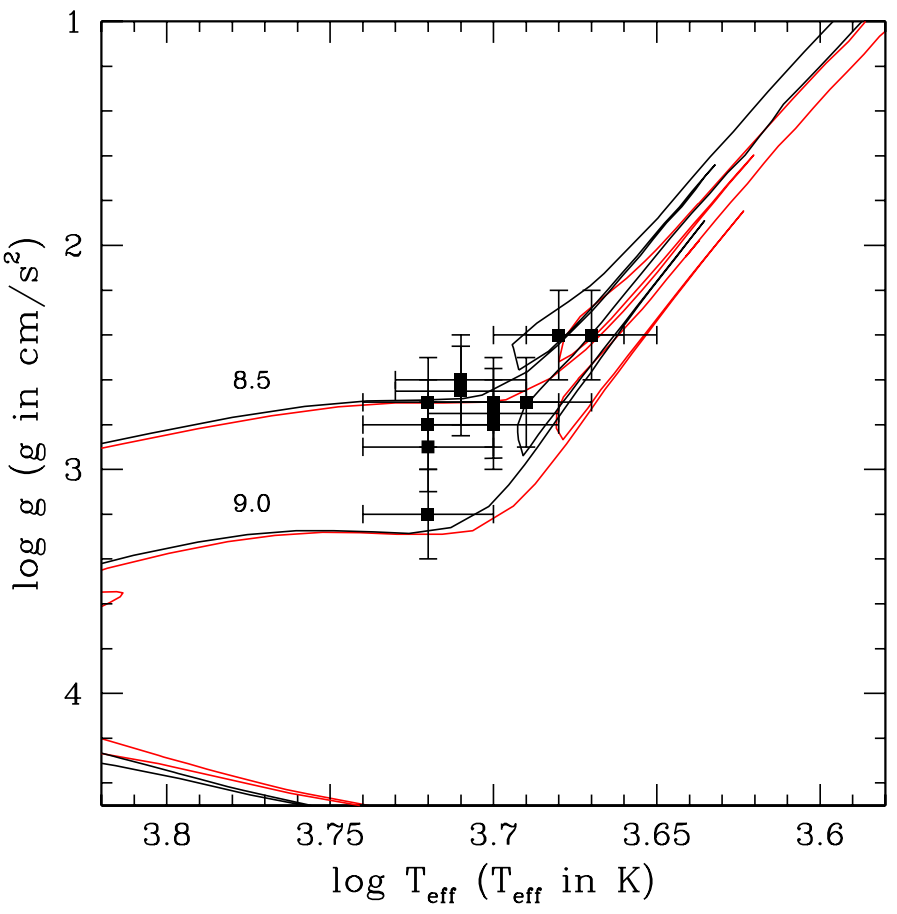

Fig. 3. Positions of the metal-rich barium stars compared with isochrones of Girardi et al. (2000). Red lines represent metallicities of $Z=0.03$ and black lines represent metallicities of $Z=0.02$. The numbers correspond to the age in units of Gyr.

Table 9. Space velocities and Galactic orbital parameters for some metal-rich barium stars.

\begin{tabular}{lccccccc}
\hline \hline Star & $\begin{array}{c}U_{\text {LSR }} \\
\mathrm{km} \mathrm{s}^{-1}\end{array}$ & $\begin{array}{c}V_{\text {LSR }} \\
\mathrm{km} \mathrm{s}^{-1}\end{array}$ & $\begin{array}{c}W_{\text {LSR }} \\
\mathrm{km} \mathrm{s}^{-1}\end{array}$ & $\begin{array}{c}R_{\min } \\
\mathrm{kpc}\end{array}$ & $\begin{array}{c}R_{\max } \\
\mathrm{kpc}\end{array}$ & $\begin{array}{c}Z_{\max } \\
\mathrm{kpc}\end{array}$ & $e$ \\
\hline HD 46040 & -2.3 & -2.2 & -15.0 & 7.19 & 7.95 & 0.3 & 0.05 \\
HD 82765 & 9.5 & -3.7 & 5.9 & 7.73 & 8.12 & 0.1 & 0.02 \\
HD 100012 & 11.8 & -5.1 & 4.1 & 7.53 & 8.10 & 0.1 & 0.04 \\
HD 130386 & -20.8 & -25.0 & 13.7 & 6.46 & 8.35 & 0.3 & 0.13 \\
HD 139660 & 5.5 & -26.0 & 4.7 & 6.47 & 8.20 & 0.1 & 0.12 \\
HD 198590 & -14.9 & 2.8 & 26.3 & 7.97 & 8.72 & 0.6 & 0.05 \\
HD 212209 & 50.6 & 20.0 & -7.0 & 7.60 & 10.37 & 0.4 & 0.15 \\
\hline
\end{tabular}

determination of radial velocity, it is interesting to evaluate the uncertainties introduced by an unknown orbital motion. For this purpose we analyzed observed orbital velocity amplitude of barium binary stars with determined orbital elements (Jorissen \& Mayor 1988; Udry et al. 1998b,a). Figure 4 shows the dependence between observed orbital velocity amplitudes and the periods of analyzed stars. The mean value of the amplitude for the whole sample was found to be $6 \mathrm{~km} \mathrm{~s}^{-1}$. This value was considered as the uncertainty in the determined radial velocities of our stars.

Inspecting Table 9 we may conclude that our sample stars come from several places of the Galaxy, with mean space velocities of $U_{\mathrm{LSR}}=6 \pm 23, V_{\mathrm{LSR}}=-6 \pm 16$ and $W_{\mathrm{LSR}}=5 \pm 13 \mathrm{~km} \mathrm{~s}^{-1}$. Their minimum and maximum distances to the Galactic center are $7.3 \pm 0.6 \mathrm{kpc}$ and $8.5 \pm 0.8 \mathrm{kpc}$, respectively. Most of the stars have $V_{\text {LSR }}$ negative results, which can be taken as indication that they lag in the Galactic rotation compared to the solar motion. Two stars, HD 198590 and HD 212209, have $V_{\text {LSR }}$ opposite velocities compared to the rest of the stars. These properties can be taken as evidence that these metal-rich barium stars as well as other metal-rich and super metal-rich stars form

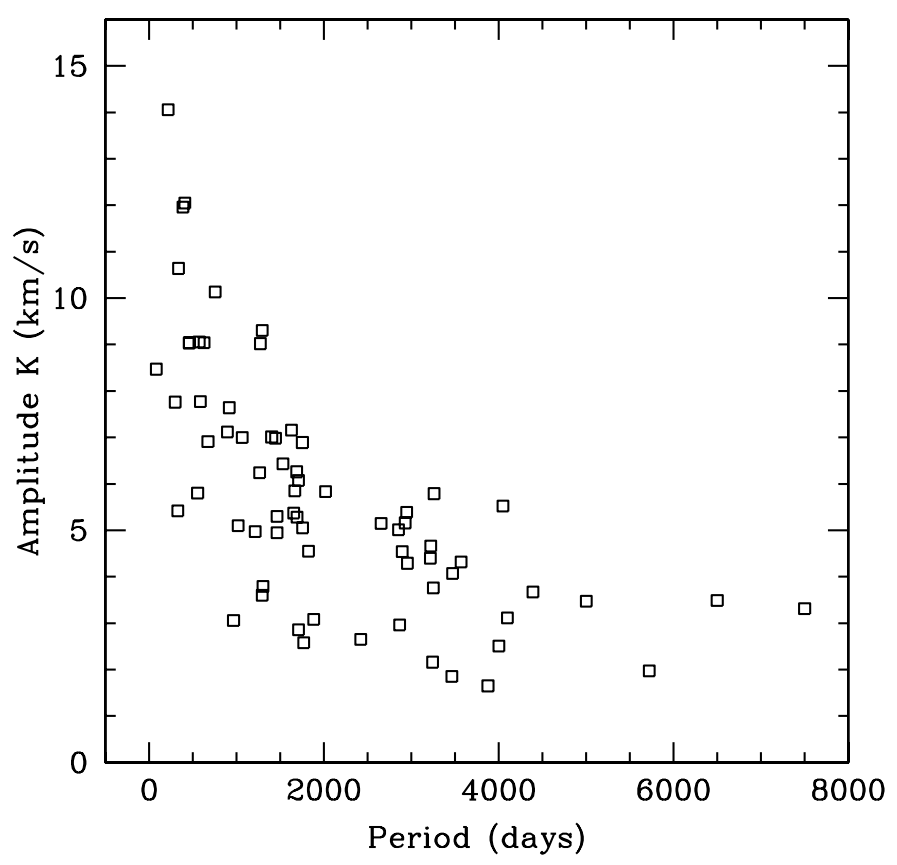

Fig. 4. Amplitude versus period for a sample of barium stars.

an inhomogeneous group. Metal-rich stars with space velocities and/or $R_{\min }$ and $R_{\max }$ that differ from each other of the same studied sample would have a different star-formation history and evolution (Pakhomov et al. 2009). It also interesting to notice that HD 212209 has the highest eccentricity among the other stars. Most of the stars have $R_{\max }$ less than $8.5 \mathrm{kpc}$, that is, within the solar circle, which seems to agree with the results obtained by Raboud et al. (1998) and Grenon (1999) for metal-rich stars. Other results may be seen in Table 9. All metal-rich barium stars lie inside in the $(U, V)$-plane of metal-rich stars of Raboud et al. (1998) and Grenon (1999), thus indicating that they display similar kinematical properties of non s-process enriched metal-rich stars. Finally, the star HD 198590 with the highest $Z_{\max }$ value among the analyzed stars, with a metallicity of $\approx+0.2$, lies in the upper limit (with very few others) of the $Z_{\max }$ value in the $Z_{\max }{ }^{-}$ metallicity diagram of Grenon (1999). In Fig. 13 of Soubiran \& Girard (2005) this star lies between the thin-disk stars at $([\mathrm{Fe} / \mathrm{H}]$, $\left.Z_{\max }\right)=(0.0,0.1-0.2 \mathrm{kpc})$ and the thick-disk stars at $([\mathrm{Fe} / \mathrm{H}]$, $\left.Z_{\max }\right) \approx(0.5,1.0 \mathrm{kpc})$. The other stars in Table 9 belong to the thin-disk stars with lower $Z_{\max }$ values.

\subsection{Abundances}

In this section we compare the abundance ratios $[\mathrm{X} / \mathrm{Fe}]$ of the metal-rich barium giants analyzed in this work with the results of some studies already made for giant stars. We used for comparison the data from local disk field giants studied by Luck \& Heiter (2007), disk red-clump giants studied by Mishenina et al. (2006, 2007), metal-rich giants studied by Pakhomov et al. (2009) and two metal-rich open clusters and $\mu$ Leo studied by Carretta et al. (2007). Figures 5 to 8 show the $[\mathrm{X} / \mathrm{Fe}]$ ratios with metallicity for the barium giants of our sample and the results for the non s-process enriched giant stars mentioned above. We will see that with respect to the sodium, aluminun, $\alpha$-elements, chromium and nickel, the abundance ratios follow the same trend as seen in the disk-giant stars. For the heavy-elements, the [s/Fe] ratio, where 's' means the mean abundance of the elements 
synthesized by s-process, ranges from +0.2 to +1.2 , thus indicating different degrees of enhancements.

\subsubsection{Sodium to nickel}

Sodium and aluminum are mainly produced by hydrostatic carbon burning in massive stars (Woosley \& Weaver 1995). Sodium and aluminun in disk stars has been observed among others by Edvardsson et al. (1993) and Feltzing \& Gustafsson (1998). Over the range $-1.0<[\mathrm{Fe} / \mathrm{H}]<0.0$, the ratios $[\mathrm{Na} / \mathrm{Fe}]$ and $[\mathrm{Al} / \mathrm{Fe}]$ are slight enhanced by $\approx 0.1 \mathrm{dex}$. For metal-rich stars Edvardsson et al. (1993) and Feltzing \& Gustafsson (1998) (see also Shi et al., 2004) observed an upturn in the $[\mathrm{Na} / \mathrm{Fe}]$ ratio for metal-rich stars, that is $[\mathrm{Na} / \mathrm{Fe}]=+0.2$ at $[\mathrm{Fe} / \mathrm{H}]=+0.2$. Shi et al. (2004) argued that to explain this Na overabundance for the metal-rich stars, large amounts of sodium have been produced by $\mathrm{NeNa}$ cycle in the interiors of AGB stars. Figure 5 shows that both the local giants and the red clump giants exhibit the same trend as seen in dwarf stars. The barium giants of our sample follow the same trend. For aluminun the ratio $[\mathrm{Al} / \mathrm{Fe}]$ is almost constant at $\approx 0.1$ in the metallicity range -2.0 to 0.2 (Carretta et al. 2002; Edvardsson et al. 1993; Feltzing \& Gustafsson 1998). The local giants and our data for the barium giants seem to follow the same trend as seen for all these previous studies.

In the thick- and thin-disk stars the $\alpha$-element abundances given by the mean of $\mathrm{Mg}, \mathrm{Si}, \mathrm{Ca}$, and $\mathrm{Ti}$ are overabundant by $\approx 0.2$ dex at $-1.0<[\mathrm{Fe} / \mathrm{H}]<-0.5$ and then decrease by $0.1-$ 0.0 dex at $-0.5<[\mathrm{Fe} / \mathrm{H}]<0.0$ (Edvardsson et al. 1993; Reddy et al. 2003, 2006). Feltzing \& Gustafsson (1998) observed that $[\alpha / \mathrm{Fe}]$ ratio has the same trend as detected by the other authors at $-0.5<[\mathrm{Fe} / \mathrm{H}]<0.0$. Up to $[\mathrm{Fe} / \mathrm{H}]=+0.3$ the $[\alpha / \mathrm{Fe}]$ ratio remains flat at $[\mathrm{Fe} / \mathrm{H}]=0.0-0.1$. The analysis of the local disk field giants studied by Luck \& Heiter (2007) and disk red-clump giants studied by Mishenina et al. (2006) showed that giants display the same trend as mentioned above for the dwarfs. Figure 6 shows that the $[\alpha / \mathrm{Fe}]$ ratio versus $[\mathrm{Fe} / \mathrm{H}]$ for the barium stars follows the same trend as the giants. The ratios $[\mathrm{X} / \mathrm{Fe}]$ for the $\alpha$-elements, $\mathrm{Mg}, \mathrm{Si}, \mathrm{Ca}$, and $\mathrm{Ti}$ are discussed below.

Magnesium (like oxygen) is produced in massive stars at $\approx 25 M_{\odot}$ as predicted by the nucleosynthesis theory (Woosley $\&$ Weaver 1995) and iron is mainly produced by SNeI events. It would be expected that both ratios, $[\mathrm{O} / \mathrm{Fe}]$ and $[\mathrm{Mg} / \mathrm{Fe}]$, would decrease with increasing metallicity. Indeed, the $[\mathrm{O} / \mathrm{Fe}]$ ratio at $[\mathrm{Fe} / \mathrm{H}]=-1.0$ is $\approx+0.4$, while at $[\mathrm{Fe} / \mathrm{H}]=0.2$ is $\approx-0.1$ (Edvardsson et al. 1993). In halo stars, the $[\mathrm{Mg} / \mathrm{Fe}]$ ratio also increases (like oxygen) toward lower metallicities (Norris et al. 2001). In dwarf disk stars, for metallicities higher than $[\mathrm{Fe} / \mathrm{H}]$ $>-0.2$ and up to +0.2 , the ratio $[\mathrm{Mg} / \mathrm{Fe}]$ becomes flat at $\approx 0.1$ (Feltzing \& Gustafsson 1998; Chen et al. 2000). This observed trend of the $[\mathrm{Mg} / \mathrm{Fe}]$ ratio toward the higher metallicities would suggest that $\mathrm{Mg}$ would not only be produced by massive stars but also by type I SNe events (Chen et al. 2000). Timmes et al. (1995) predict subsolar values for the $[\mathrm{Mg} / \mathrm{Fe}]$ ratio at higher metallicities for the evolution of the magnesium abundances in the Galaxy, which is not seen in all the analysis of the $[\mathrm{Mg} / \mathrm{Fe}]$ ratio. The analysis of the $[\mathrm{Mg} / \mathrm{Fe}]$ ratio of local giants by Luck \& Heiter (2007) as well as the barium giants of our sample shows the same trend as seen in dwarfs for metallicities higher than $[\mathrm{Fe} / \mathrm{H}]>0.0$ (Fig. 5). However, the $[\mathrm{Mg} / \mathrm{Fe}]$ ratio for the red clump giants analyzed by Mishenina et al. (2006) behaves like the $[\mathrm{O} / \mathrm{Fe}]$ ratio.

Silicon can be produced by $10-30 M_{\odot}$ stars by hydrostatic oxygen burning and also during the eventual type II supernovae explosion (Woosley \& Weaver 1986). In halo stars at
$[\mathrm{Fe} / \mathrm{H}]=-3.0$ it is enhanced by $\approx+0.5$ with larger scatter (Norris et al. 2001; Carretta et al. 2002). For dwarfs with metallicities higher than $[\mathrm{Fe} / \mathrm{H}]=0.0$, it seems that silicon behaves like magnesium and has a $[\mathrm{Si} / \mathrm{Fe}]$ ratio of $\approx 0.05$ (Edvardsson et al. 1993). Inspecting the results of Feltzing \& Gustafsson (1998), one can note that silicon flattens at a mean ratio of $[\mathrm{Si} / \mathrm{Fe}]=0.0$ for $[\mathrm{Fe} / \mathrm{H}]=0.0-0.4$. For the red clump giants, the local giants and the barium giants analyzed in this work the relative-to-iron silicon abundance flattens at a mean ratio of of $[\mathrm{Si} / \mathrm{Fe}] \approx 0.1$ for $[\mathrm{Fe} / \mathrm{H}]=0.0-0.3$.

Calcium shares the same nucleosynthesis site as silicon. In halo stars it is enhanced by $0.2-0.3$ dex (Norris et al. 2001). In dwarfs and for metallicities higher than $[\mathrm{Fe} / \mathrm{H}]>-0.2$, calcium flattens toward a mean ratio $[\mathrm{Ca} / \mathrm{Fe}]$ of $\approx 0.0$. The same trend is seen in barium and red clump giants but for the local giants, the $[\mathrm{Ca} / \mathrm{Fe}]$ ratio is lower by $-0.2 \mathrm{dex}$ at $[\mathrm{Fe} / \mathrm{H}]=+0.2($ Luck \& Heiter 2007).

Titanium is usually considered an $\alpha$-element that is produced by explosive oxygen burning but could also be produced by type Ia supernovae (Woosley \& Weaver 1995). Observationally, it behaves like an $\alpha$-element. Indeed, the [Ti/Fe] ratio for halo stars displays a similar trend as the $[\mathrm{Ca} / \mathrm{Fe}]$ ratio. In dwarfdisk stars $[\mathrm{Ti} / \mathrm{Fe}]$ slightly decreases with increasing metallicity (Edvardsson et al. 1993). For higher metallicities it seems that the ratio $[\mathrm{Ti} / \mathrm{Fe}]$ flattens around $\approx 0.1$. In the present work, the $[\mathrm{Ti} / \mathrm{Fe}]$ ratios are usually lower than the $[\mathrm{Mg}, \mathrm{Si}, \mathrm{Ca} / \mathrm{Fe}]$ ratios, displaying a similar trend as local-disk giants (Luck \& Heiter 2007).

The iron peak elements are formed in large amounts in type Ia supernovae and all its members should follow the same trend with iron abundance, therefore the $[\mathrm{X} / \mathrm{Fe}]$ ratios should remain constant within the range of metallicity studied. Indeed, nickel, does remain constant with $[\mathrm{Ni} / \mathrm{Fe}]=0.0$ for $-0.8<$ $[\mathrm{Fe} / \mathrm{H}]<0.1$. Luck \& Heiter (2007) also found a tight correlation between $[\mathrm{Fe} / \mathrm{H}]$ and $[\mathrm{Ni} / \mathrm{H}]$ with a slope of 1.04 also between $-0.8<[\mathrm{Fe} / \mathrm{H}]<0.1$. However, some studies show a possible upturn after $[\mathrm{Fe} / \mathrm{H}]>0.1$ for red clump giants (Mishenina et al. 2006; Liu et al. 2007) and dwarfs (Chen et al. 2000). This upturn is seen in Luck \& Heiter's data and also in Fig. 4 of Soubiran \& Girard (2005). For the element chromium, the study for the local giants of Luck \& Heiter (2007) found a tight correlation between $[\mathrm{Fe} / \mathrm{H}]$ and $[\mathrm{Cr} / \mathrm{H}]$ with a slope of 1.06 , that is $[\mathrm{Cr} / \mathrm{Fe}]=0.0$ for $-0.8<[\mathrm{Fe} / \mathrm{H}]<0.2$, despite some discrepant points, as they reported. The barium stars of our sample also follow the general trend seen for the field giants, for the ratios of $[\mathrm{Ni} / \mathrm{Fe}]$ and $[\mathrm{Cr} / \mathrm{Fe}]$.

\subsection{2. s-process elements: $\mathrm{Y}, \mathrm{Zr}$, La, Ce and $\mathrm{Nd}$}

The main site of $s$-process elements (and also carbon) production are low-mass AGB stars $\left(M \leq 3.0 M_{\odot}\right)$ during the thermally pulsing phase (TP-AGB). Nucleosynthesis of s-process takes place in the $\mathrm{C}$-rich intershell convective zone, the zone between the H-burning shell and the He-burning shell, of which the source of neutrons for the s-process to occur is the ${ }^{13} \mathrm{C}(\alpha, \mathrm{n}){ }^{16} \mathrm{O}$ reaction (Lattanzio \& Wood 2003). Figure 8 shows the ratio [s/Fe], where "s" represents the mean of the elements created by slow neutron capture reactions (s-process): Y, Zr, La, Ce, and Nd. As we can see from Fig. 8, all stars except HD 100012 are enhanced in the s-process elements when compared to the same ratio of the field stars. HD 100012 has a [s/Fe] ratio of +0.22 and therefore cannot be considered as a barium star because some stars at this metallicity have a similar $[\mathrm{s} / \mathrm{Fe}]$ ratio as $\mathrm{HD} 157919$ with $[\mathrm{s} / \mathrm{Fe}]=+0.2$ at $[\mathrm{Fe} / \mathrm{H}]=+0.14$. Among the 


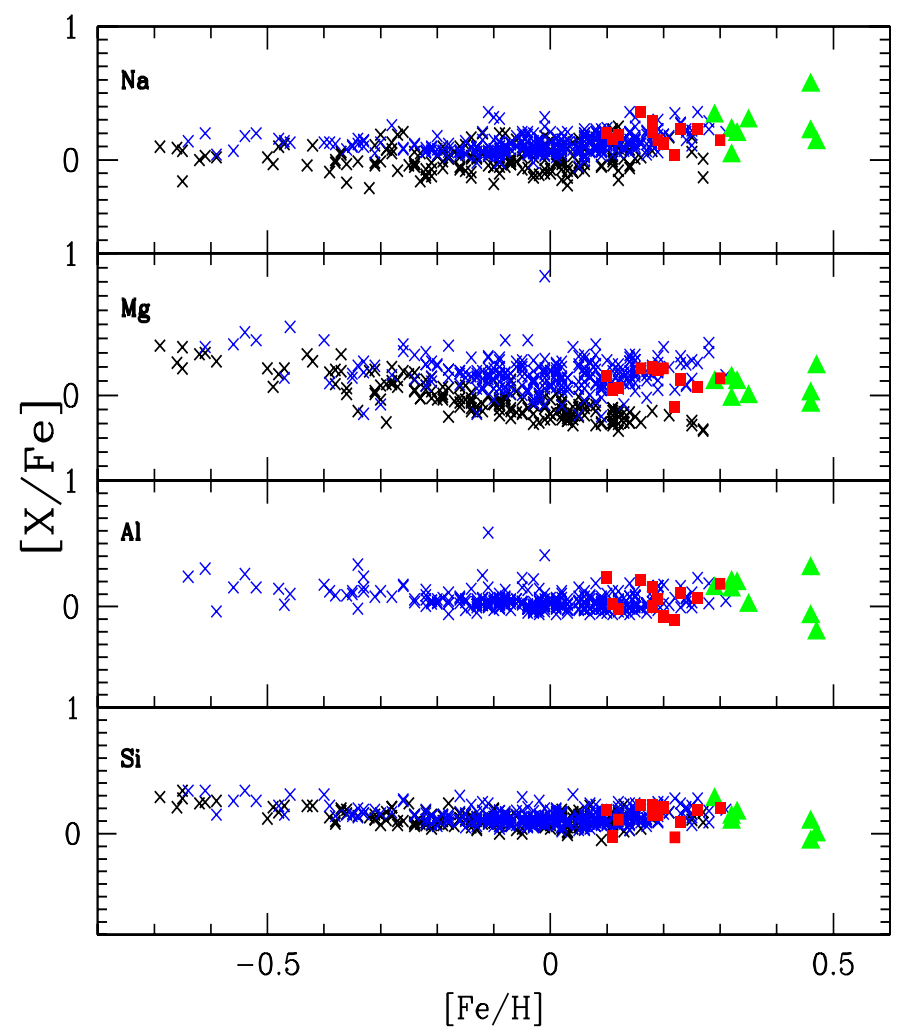

Fig. 5. Abundance ratios $[\mathrm{X} / \mathrm{Fe}]$ vs. $[\mathrm{Fe} / \mathrm{H}]$. Metal-rich barium stars red squares; field giants of Luck \& Heiter (2007), blue crosses; clump giants of Mishenina et al. (2006), black crosses; metal-rich giants stars and two metal-rich open clusters and $\mu$ Leo studied by Pakhomov et al. (2009) and Carretta et al. (2007), respectively, green triangles.

local giants analyzed by Luck \& Heiter (2007), only one barium star was spotted, HD 104979 (Zacs 1994), which can be seen at $[\mathrm{Fe} / \mathrm{H}]=-0.33$ with $[\mathrm{s} / \mathrm{Fe}]=+0.54$.

Figure 8 also shows that our sample stars differs from the field stars only by the degree of s-process enrichment observed in their atmospheres. Models of galactic chemical evolution do not predict this enrichment at this metallicity (Travaglio et al. 1999, 2004). Therefore, the atmospheres of these stars were contaminated either through the stellar evolution or an extrinsic event that may have happened in the past, i.e., mass-transfer hypothesis. The first hypothesis can be ruled out because of their position in the $\log g-\log T_{\text {eff }}$ diagram (Fig. 2). Finally, Fig. 8 also shows that the abundance of zirconium is poorly investigated in normal giant stars in this metallicity range.

Figure 9 shows the $[\mathrm{s} / \mathrm{Fe}]$ ratios for chemically peculiar binary systems including not only the barium stars (giants and dwarfs) but also the yellow symbiotic stars, the $\mathrm{CH}$ stars, and the CEMP (carbon enhanced metal-poor) stars, those that have already been proved as binary systems. The definition of "s" varies from author to author and in some cases depends on the quality and/or on the wavelength range of the available spectra. Among CEMP stars, there are six that are binaries: CS 22942-019, CS 22948-027, CS 29497-030, CS 29497-034, CS 22964-161, and HE 0024-2523, the data of which concerning their carbon and heavy-element $(Z>56)$ overabundances and binarity were taken from the results of Preston \& Sneden (2001); Sivarani et al. (2004); Barbuy et al. (2005); Lucatello et al. (2003); Thompson et al. (2008); Aoki et al. (2002) and

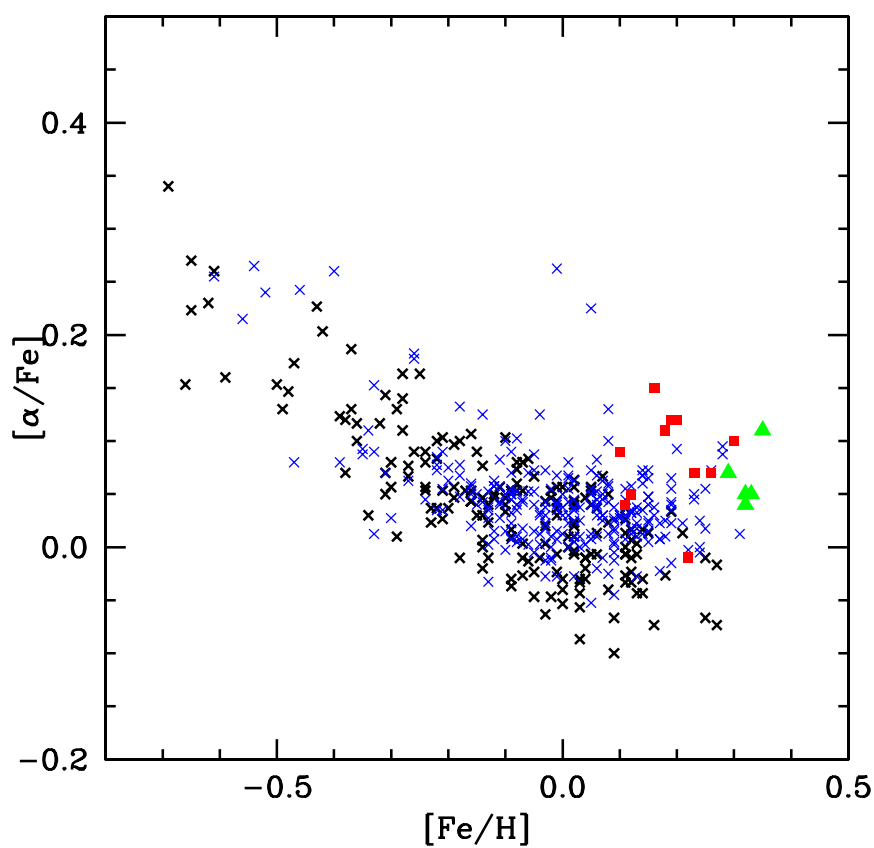

Fig. 6. Abundance ratios $[\alpha / \mathrm{Fe}]$ vs. $[\mathrm{Fe} / \mathrm{H}]$. Symbols have the same meaning as in Fig. 5.

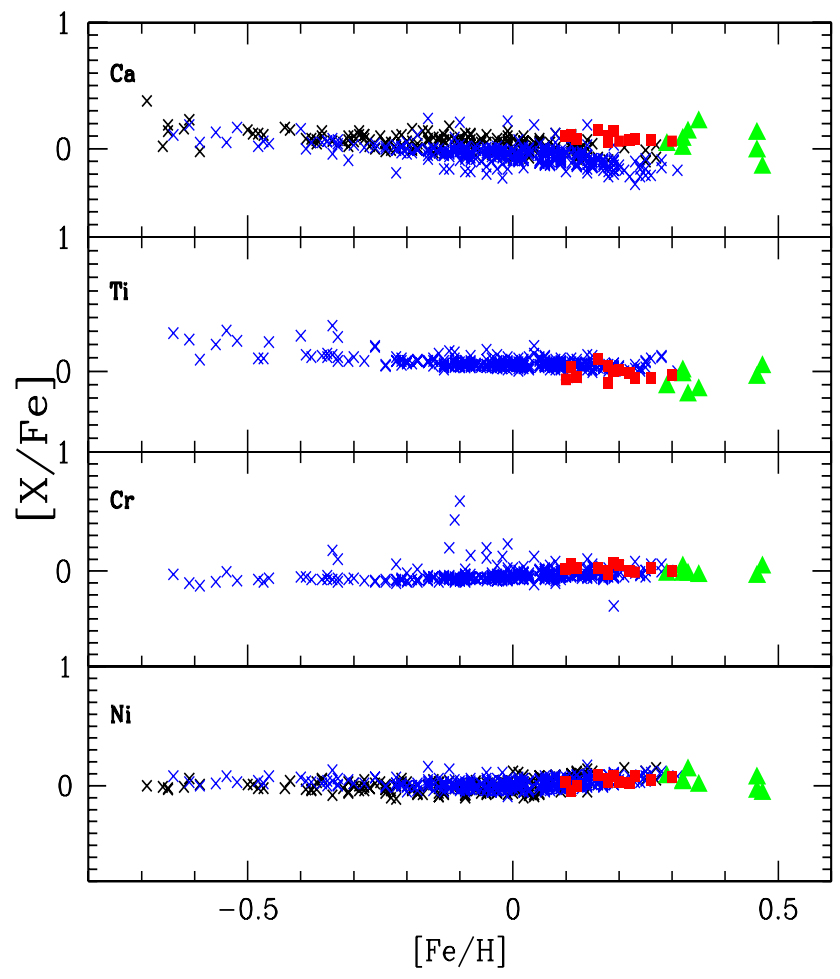

Fig. 7. Abundance ratios $[\mathrm{X} / \mathrm{Fe}]$ vs. $[\mathrm{Fe} / \mathrm{H}]$. Symbols have the same meaning as in Fig. 5.

Hill et al. (2000). According to the mass-transfer hyphotesis for the origin of the barium stars, one of the binary components ejects its envelope that is enriched in the s-process elements produced during the AGB phase, and this matter falls onto the companion, which in turn becomes enriched in these elements, and now is the barium star. The different ratios $[\mathrm{s} / \mathrm{Fe}]$ observed 


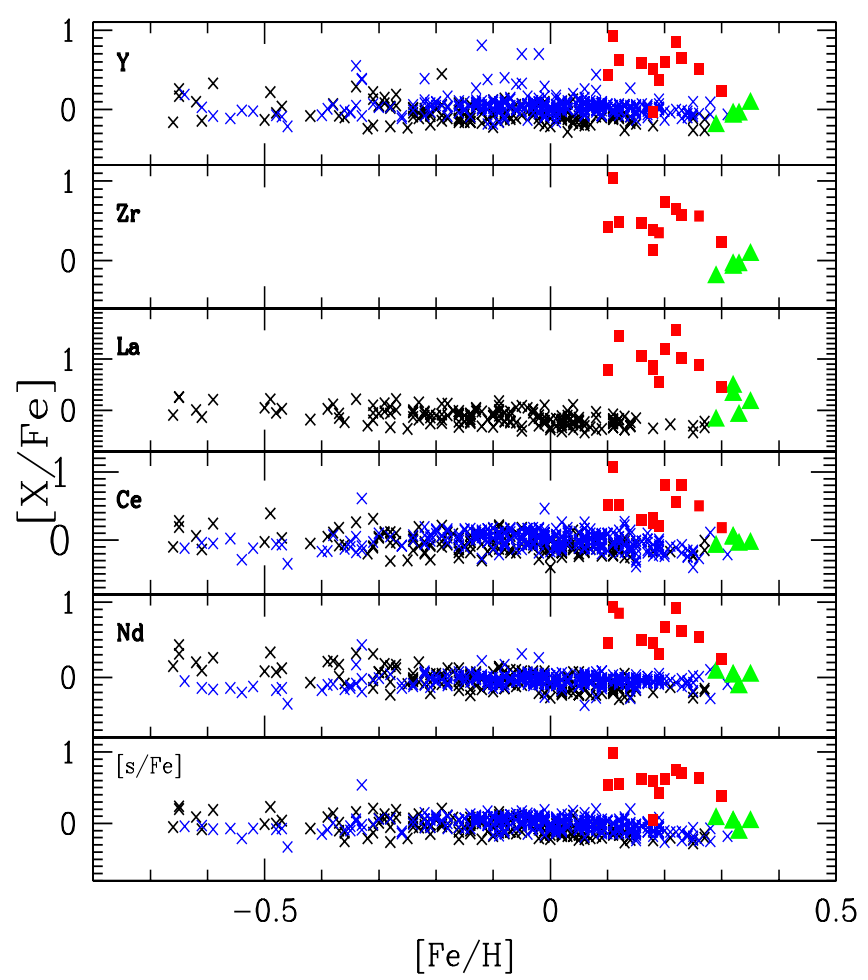

Fig. 8. Abundance ratios $[\mathrm{X} / \mathrm{Fe}]$ vs. $[\mathrm{Fe} / \mathrm{H}]$. Symbols have the same meaning as in Fig. 5. Clump giants are from Mishenina et al. (2007).

among the barium stars, also seen in this study, depend on the material received by the companion as well as whether this material is partially or fully mixed in the atmosphere of the barium stars. Theoretical studies for the production of the s-process elements in intrinsic AGB stars (Gallino et al. 1998; Busso et al. 1999) indicate that there is a trend that the s-process elements are more easily produced at lower metallicities as a result of the operation of the reaction ${ }^{13} \mathrm{C}(\alpha, \mathrm{n}){ }^{16} \mathrm{O}$ as a neutron source. When several classes of chemically-peculiar stars are examined all together (Fig. 9), this phenomenon is observed.

According to Busso et al. (1999) and Travaglio et al. (2004), the neutron-capture nucleosynthesis in AGB stars is metallicitydependent. Among the elements created by the s-process nucleosynthesis, the first neutron magic peak elements (such as $\mathrm{Y}$ and $\mathrm{Zr}$ ) are the dominant products of the neutron captures in AGB models at metallicities around $[\mathrm{Fe} / \mathrm{H}]=-0.4$. At lower metallicities, $[\mathrm{Fe} / \mathrm{H}] \approx-1.0$, the second neutron magic peak elements (Ba-La-Ce-Nd) are the dominant. The origin of these two peaks is the result of the neutron magic numbers 50 and 82 nuclei, which have smaller absorption cross-sections than their neighbor nuclei and therefore turn out to be more stable than others. Owing to this, it is possible to monitor the s-process efficiency through the relative abundances of the second-peak elements to the first-peak elements. In this way the s-elements at the secondpeak are usually referred to as the "heavy" s-process elements, in our case, $\mathrm{La}, \mathrm{Ce}$, and $\mathrm{Nd}$, while s-elements at the first-peak are usually referred to as the "light" elements, in our case, Y and Zr. Thus, the average logarithmic ratio [hs/ls] has been widely used to measure the efficiency of neutron captures. Table 10 provides the ratios $[\mathrm{ls} / \mathrm{Fe}],[\mathrm{hs} / \mathrm{Fe}]$ as well as the ratio [hs/ls] for the studied stars. Inspecting Table 10, we see that our derived values for the indices [hs/ls], [hs/Fe], [ls/Fe] for all metal-rich barium stars, except for HD 46040, are in the range, -0.3 to $0.0,0.2$ to 0.7

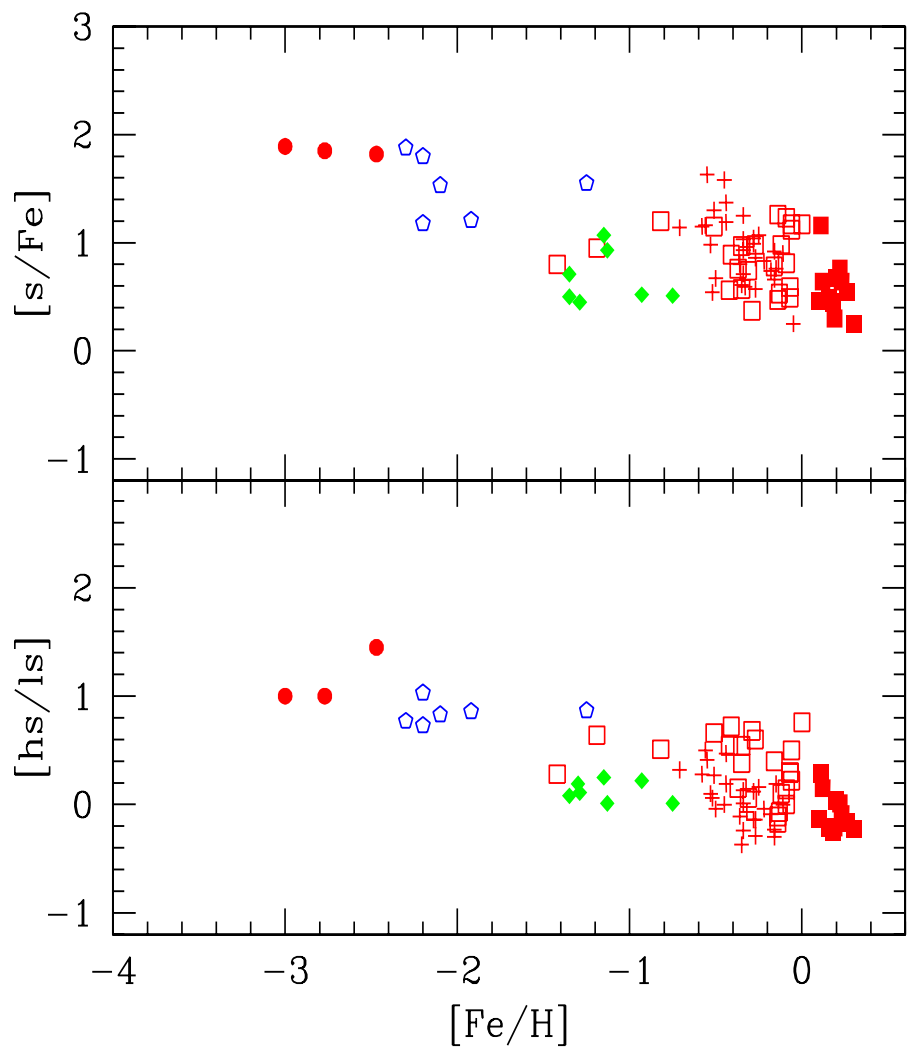

Fig. 9. Diagram of $[\mathrm{s} / \mathrm{Fe}]$ versus $[\mathrm{Fe} / \mathrm{H}]($ top $)$ and $[\mathrm{hs} / \mathrm{ls}]$ versus $[\mathrm{Fe} / \mathrm{H}]$ (bottom) for several classes of chemically peculiar binary stars. Metalrich barium stars (filled red squares); barium giants previously analyzed (red open squares); barium dwarfs (plus red crosses); $\mathrm{CH}$ stars (blue open polygons); yellow symbiotic (green symbols) and CEMP-s stars which are members of binary systems (red filled circles).

Table 10. Indices of the s-process.

\begin{tabular}{lccc}
\hline \hline star & {$[\mathrm{ls} / \mathrm{Fe}]$} & {$[\mathrm{hs} / \mathrm{Fe}]$} & {$[\mathrm{hs} / \mathrm{ls}]$} \\
\hline CD-25 6606 & 0.55 & 0.70 & 0.15 \\
HD 46040 & 0.99 & 1.28 & 0.29 \\
HD 49841 & 0.75 & 0.76 & 0.01 \\
HD 82765 & 0.43 & 0.22 & -0.21 \\
HD 84734 & 0.62 & 0.66 & 0.04 \\
HD 85205 & 0.70 & 0.61 & -0.09 \\
HD 101079 & 0.54 & 0.41 & -0.13 \\
HD 130386 & 0.62 & 0.41 & -0.21 \\
HD 139660 & 0.64 & 0.47 & -0.17 \\
HD 198590 & 0.60 & 0.35 & -0.25 \\
HD 212209 & 0.39 & 0.16 & -0.23 \\
\hline
\end{tabular}

and 0.4 to 0.8 . These values seem to agree well when compared with model predictions given by Busso et al. (2001) as seen in their Figs. 7-9 for the ratios [hs/ls], [hs/Fe], [ls/Fe] vs. metallicity for the standard case (ST/1.5). The ST case corresponds to $4 \times 10^{-6} M_{\odot}$ of ${ }^{13} \mathrm{C}$ for AGB stars between 1.5 and $3.0 M_{\odot}$ for a metallicity of $[\mathrm{Fe} / \mathrm{H}]=-0.3$, which accounts for the main component of the s-process in solar system (Busso et al. 2001). HD 46040 has higher values for the indices [hs/ls] and [hs/Fe] for a star at this metallicity and its $[1 \mathrm{~s} / \mathrm{Fe}]$ index marginally falls in the limit of the model predictions.

In Fig. 9 we show the [hs/ls] index as a function of the metallicity for the same classes of chemically peculiar binary systems. As for the $[\mathrm{s} / \mathrm{Fe}]$ ratio vs metallicity, the $[\mathrm{hs} / \mathrm{ls}]$ index also 
increases toward lower metallicities, with a large spread. In both figures we can see that the position of metal-rich barium stars are useful targets for constraining models for the nucleosynthesis of the s-process at higher metallicities.

\section{Conclusions}

Our abundance analysis has been performed by employing highresolution optical spectra of a sample of metal-rich barium stars with the aim to obtain their abundance pattern and kinematical properties, and it can be summarized as follows:

1. The stars have enhancement factors, $[\mathrm{s} / \mathrm{Fe}]$, from 0.25 up to 1.16. These overabundances are expected for stars at this metallicity according to the models of Busso et al. (2001), which are based on the observed indices $[1 \mathrm{~s} / \mathrm{Fe}],[\mathrm{hs} / \mathrm{Fe}]$ and [hs/ls]. The abundance pattern of the iron peak elements, of the elements of the $\alpha$ group, as well as that of sodium and aluminum follows the disk abundance. The [alpha/Fe] ratios in our stars do not show any trace of overabundances, while bulge stars display increased ratios (Lecureur et al. 2007). Therefore, these metal-rich barium stars do not belong to the bulge population. It is probable that like the most of the non s-process enriched metal-rich stars, the barium stars analyzed in this work belong to the thin-disk stars, as can be deduced form their low $Z_{\max }$ values. Also interesting to note is that all stars analyzed in this work have low values for the eccentricities, which indicates almost circular orbits in agreement with this characteristic of the other non s-process enriched metal-rich stars previously analyzed (Pakhomov et al. 2009; Gratton \& Sneden 1990) and which agrees with the results of Raboud et al. (1998).

2. Considering evolutionary aspects, metal-rich barium stars put another constraint on the evolution of barium dwarfs to barium giants. Because all $\mathrm{CH}$ subgiants and barium dwarfs analyzed so far have metallicities less than the metallicity of the Sun, except those very few subgiants analyzed in this paper, metal-rich barium dwarfs/subgiants are yet to be found. In this respect the same question can be addressed to the yellow symbiotics. All yellow symbiotics, except for the D'-type, which probably are not symbiotic stars (Corradi \& Schwarz 1997; Pereira 2005), are halo objects and their connection with metal-poor barium stars have been investigated in the recent years (Smith et al. 1996, 1997; Jorissen et al. 2005; Pereira \& Roig 2009). Consequently, disk yellow symbiotics at near solar metallicity have not yet been identified.

3. Metal-rich barium stars are useful objects for investigating the heavy-element abundance pattern at metalicities higher than the metallicity of the Sun because they are free of many molecular features that are usually seen in the spectra of MS, $\mathrm{S}$, and carbon stars. The barium star phenomenon once it extend toward higher metallicities, shares several properties with the non s-process enriched metal-rich and super metalrich giant stars previously investigated.

Acknowledgements. This research has made use of the SIMBAD database, operated at CDS, Strasbourg, France. We thank our referee for detailed comments that have improved the presentation of the present work.

\section{References}

Allen, D. M., \& Barbuy, B. 2006, A\&A, 454, 917

Alonso, A., Arribas, S., \& Martínez-Roger, C. 1999, A\&AS, 140, 261
Antipova, L. I., Boyarchuk, A. A., Pakhomov, Y. V., \& Panchuk, V. E. 2003, Astron. Rep., 47, 648

Antipova, L. I., Boyarchuk, A. A., Pakhomov, Y. V., \& Panchuk, V. E. 2004, Astron. Rep., 48, 597

Aoki, W., Ryan, S. G., Norris, J. E., et al. 2002, ApJ, 580, 1149

Arp, H. 1965, ApJ, 141, 43

Barbuy, B., \& Grenon, M. 1990, in European Southern Observatory Conference and Workshop Proceedings, ed. B. J. Jarvis, \& D. M. Terndrup, 35, 83

Barbuy, B., Spite, M., Spite, F., et al. 2005, A\&A, 429, 1031

Bessell, M. S., Castelli, F., \& Plez, B. 1998, A\&A, 333, 231

Bidelman, W. P. 1981, AJ, 86, 553

Biemont, E., Grevesse, N., Hannaford, P., \& Lowe, R. M. 1981, ApJ, 248, 867

Busso, M., Gallino, R., \& Wasserburg, G. J. 1999, ARA\&A, 37, 239

Busso, M., Gallino, R., Lambert, D. L., Travaglio, C., \& Smith, V. V. 2001, ApJ, 557,802

Carretta, E., Gratton, R., Cohen, J. G., Beers, T. C., \& Christlieb, N. 2002, AJ, 124,481

Carretta, E., Bragaglia, A., \& Gratton, R. G. 2007, A\&A, 473, 129

Castro, S., Rich, R. M., Grenon, M., Barbuy, B., \& McCarthy, J. K. 1997, AJ, 114,376

Cayrel, R. 1988, in The Impact of Very High S/N Spectroscopy on Stellar Physics, ed. G. Cayrel de Strobel, \& M. Spite, IAU Symp., 132, 345

Chen, Y. Q., Nissen, P. E., Zhao, G., Zhang, H. W., \& Benoni, T. 2000, A\&AS, 141,491

Corradi, R., \& Schwarz, H. E. 1997, in Physical Processes in Symbiotic

Drake, N. A., \& Pereira, C. B. 2008, AJ, 135, 1070

Drake, J. J., \& Smith, G. 1991, MNRAS, 250, 89

Edvardsson, B., Andersen, J., Gustafsson, B., et al. 1993, A\&A, 275, 101

Eggen, O. J. 1993, AJ, 106, 80

Feltzing, S., \& Gustafsson, B. 1998, A\&AS, 129, 237

Gallino, R., Arlandini, C., Busso, M., et al. 1998, ApJ, 497, 388

Girardi, L., Bressan, A., Bertelli, G., \& Chiosi, C. 2000, A\&AS, 141, 371

Gomez, A. E., Luri, X., Grenier, S., et al. 1997, A\&A, 319, 881

Gratton, R. G., \& Sneden, C. 1988, A\&A, 204, 193

Gratton, R. G., \& Sneden, C. 1990, A\&A, 234, 366

Grenon, M. 1972, in Age des Etoiles, ed. G. Cayrel de Strobel, \& A. M. Delplace, IAU Colloq., 17, 55

Grenon, M. 1999, Ap\&SS, 265, 331

Han, Z., Eggleton, P. P., Podsiadlowski, P., \& Tout, C. A. 1995, MNRAS, 277, 1443

Hannaford, P., Lowe, R. M., Grevesse, N., Biemont, E., \& Whaling, W. 1982, ApJ, 261, 736

Hill, V., Andrievsky, S., \& Spite, M. 1995, A\&A, 293, 347

Hill, V., Barbuy, B., Spite, M., et al. 2000, A\&A, 353, 557

Johnson, D. R. H., \& Soderblom, D. R. 1987, AJ, 93, 864

Jorissen, A., \& Mayor, M. 1988, A\&A, 198, 187

Jorissen, A., Začs, L., Udry, S., Lindgren, H., \& Musaev, F. A. 2005, A\&A, 441, 1135

Junqueira, S., \& Pereira, C. B. 2001, AJ, 122, 360

Kaufer, A., Stahl, O., Tubbesing, S., et al. 1999, The Messenger, 95, 8

Lambert, D. L., Heath, J. E., Lemke, M., \& Drake, J. 1996, ApJS, 103, 183

Lattanzio, J. C., \& Wood, P. R. 2003, Evolution, Nucleosynthesis and Pulsations of AGB stars, ed. H. J. Habing, \& H. Olofsson

Lecureur, A., Hill, V., Zoccali, M., et al. 2007, A\&A, 465, 799

Liu, Y. J., Zhao, G., Shi, J. R., Pietrzyński, G., \& Gieren, W. 2007, MNRAS, 382,553

Lucatello, S., Gratton, R., Cohen, J. G., et al. 2003, AJ, 125, 875

Luck, R. E., \& Bond, H. E. 1991, ApJS, 77, 515

Luck, R. E., \& Heiter, U. 2007, AJ, 133, 2464

MacConnell, D. J., Frye, R. L., \& Upgren, A. R. 1972, AJ, 77, 384

Martin, G. A., Fuhr, J. R., \& Wiese, W. L. 1988, Atomic transition probabilities, Scandium through Manganese, ed. G. A. Martin, J. R., Fuhr, \& W. L. Wiese

Martin, W., Fuhr, J., Kelleher, D., et al. 2002, NIST Atomic Spectra Database Version2.0 (Online Available), NIST Standard Reference Database, National Institute of Standards and Technology, Gaithersburg Maryland

Matteucci, F. 2003, The Chemical Evolution of the Galaxy, ed. F. Matteucci

McWilliam, A., \& Rich, R. M. 1994, ApJS, 91, 749

Mennessier, M. O., Luri, X., Figueras, F., et al. 1997, A\&A, 326, 722

Mishenina, T. V., Bienaymé, O., Gorbaneva, T. I., et al. 2006, A\&A, 456, 1109

Mishenina, T. V., Gorbaneva, T. I., Bienaymé, O., et al. 2007, Astron. Rep., 51, 382

Norris, J. E., Ryan, S. G., \& Beers, T. C. 2001, ApJ, 561, 1034

Pakhomov, Y. V., Antipova, L. I., Boyarchuk, A. A., Zhao, G., \& Liang, Y. 2009, Astron. Rep., 53, 685

Pereira, C. B. 2005, AJ, 129, 2469

Pereira, C. B., \& Drake, N. A. 2009, A\&A, 496, 791

Pereira, C. B., \& Drake, N. A. 2011, AJ, 141, 79

Pereira, C. B., \& Roig, F. 2009, AJ, 137, 118 
Perryman, M. A. C., \& ESA, eds. 1997, The HIPPARCOS and TYCHO catalogues, Astrometric and photometric star catalogues derived from the ESA HIPPARCOS Space Astrometry Mission, ESA Spec. Pub., 1200

Preston, G. W., \& Sneden, C. 2001, AJ, 122, 1545

Raboud, D., Grenon, M., Martinet, L., Fux, R., \& Udry, S. 1998, A\&A, 335, L61

Reddy, B. E., Tomkin, J., Lambert, D. L., \& Allende Prieto, C. 2003, MNRAS, 340,304

Reddy, B. E., Lambert, D. L., \& Allende Prieto, C. 2006, MNRAS, 367, 1329

Reyniers, M., Van Winckel, H., Gallino, R., \& Straniero, O. 2004, A\&A, 417, 269

Schaerer, D., Charbonnel, C., Meynet, G., Maeder, A., \& Schaller, G. 1993, A\&AS, 102, 339

Schönrich, R., Binney, J., \& Dehnen, W. 2010, MNRAS, 403, 1829

Shi, J. R., Gehren, T., \& Zhao, G. 2004, A\&A, 423, 683

Sivarani, T., Bonifacio, P., Molaro, P., et al. 2004, A\&A, 413, 1073

Smiljanic, R., Porto de Mello, G. F., \& da Silva, L. 2007, A\&A, 468, 679

Smith, V. V., \& Suntzeff, N. B. 1987, AJ, 93, 359

Smith, G., Edvardsson, B., \& Frisk, U. 1986, A\&A, 165, 126

Smith, V. V., Coleman, H., \& Lambert, D. L. 1993, ApJ, 417, 287
Smith, V. V., Cunha, K., Jorissen, A., \& Boffin, H. M. J. 1996, A\&A, 315, 179 Smith, V. V., Cunha, K., Jorissen, A., \& Boffin, H. M. J. 1997, A\&A, 324, 97 Smith, V. V., Pereira, C. B., \& Cunha, K. 2001, ApJ, 556, L55

Sneden, C., McWilliam, A., Preston, G. W., et al. 1996, ApJ, 467, 819

Sneden, C. A. 1973, Ph.D. Thesis, The University of Texas at Austin

Soubiran, C., \& Girard, P. 2005, A\&A, 438, 139

Spinrad, H., \& Taylor, B. J. 1969, ApJ, 157, 1279

Thompson, I. B., Ivans, I. I., Bisterzo, S., et al. 2008, ApJ, 677, 556

Timmes, F. X., Woosley, S. E., \& Weaver, T. A. 1995, ApJS, 98, 617

Travaglio, C., Galli, D., Gallino, R., et al. 1999, ApJ, 521, 691

Travaglio, C., Gallino, R., Arnone, E., et al. 2004, ApJ, 601, 864

Udry, S., Jorissen, A., Mayor, M., \& Van Eck, S. 1998a, A\&AS, 131, 25

Udry, S., Mayor, M., Van Eck, S., et al. 1998b, A\&AS, 131, 43

Van Winckel, H., \& Reyniers, M. 2000, A\&A, 354, 135

Wiese, W. L., Smith, M. W., \& Miles, B. M. 1969, Atomic transition probabilities, 2: Sodium through Calcium, A critical data compilation, ed. W. L. Wiese, M. W. Smith, \& B. M. Miles

Woosley, S. E., \& Weaver, T. A. 1986, ARA\&A, 24, 205

Woosley, S. E., \& Weaver, T. A. 1995, ApJS, 101, 181

Zacs, L. 1994, A\&A, 283, 937 